\title{
Low-Frequency Scattering by Rectangular Dielectric Particles
}

\author{
D. F. Herrick and T. B. A. Senior \\ Radiation Laboratory, The University of Michigan, Ann Arbor, MI 48109, USA \\ Received 28 June 1976/Accepted 6 December 1976
}

\begin{abstract}
The field scattered by a homogeneous isotropic dielectric particle illuminated by a low-frequency plane electromagnetic wave is expressed in terms of a single polarizability tensor which is a function of only the geometry of the particle and a material parameter $\tau$ representing either the relative permittivity or permeability of the dielectric. The mathematical formulation is specialized to the case of a rectangular parallelepiped and numerical techniques are developed for computing the tensor elements. Specific data are presented for the tensor elements of rectangular parallelepipeds having square cross sections and are compared to the results obtained for spheroids and right circular cylinders of similar dimensions.
\end{abstract}

PACS Codes: $77.20,84$

A knowledge of the electromagnetic scattering characteristics of small dielectric bodies is important in many areas of physics. The present investigation of the scattering of visible and infrared radiation by clouds and by polluted atmospheres was motivated by a consideration of the radiation balance of the earth and is also important at microwave frequencies in connection with radar meteorology from space. Techniques for computing the reflectivity and transmissivity of high altitude clouds and other aerosol-laden atmospheres have been summarized by Plass et al. [1], and an essential ingredient in all of them is the scattering from a single aerosol particle. In most theories to date the particles have been modelled as spheres, enabling the simple Mie theory to be applied, but, as noted by Kattewar and Plass [2], the spherical model introduces unknown errors in the computation of the radiation transfer. In particular, the polarization characteristics of the scattered fields are poorly simulated by Mie theory and it is therefore appropriate to develop techniques for computing the scattering from the specific particle shapes which may be present.
One frequency range where this is feasible is the low frequency or Rayleigh region where the wavelength of the radiation is much greater than the maximum linear dimension of the particle, and the present paper is directed entirely at the scattering from a single homogeneous isotropic dielectric particle at these frequencies. The far-zone scattered fields may then be attributed to induced electric and magnetic dipoles. The dependence of the scattered fields on the direction and polarization of the incident radiation is made explicit by writing the dipole moments in terms of a general polarizability tensor which is a function only of the geometry and material of the scatterer. The tensor elements are expressed as weighted surface integrals of certain potential functions and integral equations for the determination of these potentials are given.

Aerosol particles having a crystalline structure are frequently encountered, and we have chosen as a simple example the geometry of a rectangular parallelepiped. When the mathematical formulation is specialized to this particular case, it is found that the integral equations are nonsingular and their numerical solution 
is relatively straightforward. Specific data are presented for rectangular parallelepipeds of square cross section having various length-to-width ratios and (real) permittivities. These are compared with the results obtained for spheroids and right circular cylinders of similar dimensions.

\section{Mathematical Formulation}

Let $\mathrm{B}$ be the finite closed surface of a homogeneous isotropic dielectric body of volume $\mathrm{V}$ immersed in free space. The electric and magnetic field vectors of a linearly polarized plane electromagnetic wave incident on $\mathrm{B}$ are taken as

$\boldsymbol{E}^{i}=\hat{\boldsymbol{a}} \exp \left(\mathrm{i} k_{0} \hat{\boldsymbol{k}} \cdot \boldsymbol{r}\right), \quad \boldsymbol{H}^{i}=Y_{0} \hat{\boldsymbol{b}} \exp \left(\mathrm{i} k_{0} \hat{\boldsymbol{k}} \cdot \boldsymbol{r}\right)$,

where $\hat{\boldsymbol{k}}, \hat{\boldsymbol{a}}$, and $\hat{\boldsymbol{b}}$ are unit vectors specifying the directions of incidence, the electric field and the magnetic field respectively. The permittivity and permeability of free space are $\varepsilon_{0}$ and $\mu_{0}$, respectively, $k_{0}$ is the free space propagation constant and $Y_{0}=Z_{0}^{-1}$ is the intrinsic admittance. Mks units are employed and a time factor $\exp (-\mathrm{i} \omega t)$ suppressed.

For sufficiently small $k_{0}$, the scattered fields may be expanded as power series in $k_{0}$ of the form

$\boldsymbol{E}^{s}=\sum_{m=0}^{\infty}\left(\mathrm{i} k_{0}\right)^{m} \boldsymbol{E}_{m}^{s}, \quad \boldsymbol{H}^{s}=\sum_{m=0}^{\infty}\left(\mathrm{i} k_{0}\right)^{m} \boldsymbol{H}_{m}^{s}$.

How small $k_{0}$ must be to ensure convergence of the series outside a sphere of radius $r_{1}$ will depend on both the maximum linear dimension $d$ of the scatterer and the distance $r$ to the field point. From the information which is presently available, primarily for perfectly conducting bodies, the zeroth-order terms of the lowfrequency expansions appear to provide an adequate approximation to the far zone $(r \rightarrow \infty)$ scattered fields when $k_{0} d \lesssim 1 / 3$. It is well known [3] that these terms can be attributed to radiating electric and magnetic dipoles of moments $\boldsymbol{p}$ and $\boldsymbol{m}$, respectively, located at the origin of coordinates in the vicinity of the body. The expressions for the far-zone scattered fields are then

$$
\begin{aligned}
\boldsymbol{E}^{\mathrm{S}}(\boldsymbol{r})= & -\exp \left(\mathrm{i} k_{0} r\right)\left(k_{0}^{2} / 4 \pi r\right) \\
& \cdot\left[\varepsilon_{0}^{-1} \hat{\boldsymbol{r}} \times(\hat{\boldsymbol{r}} \times \boldsymbol{p})+Z_{0} \hat{\boldsymbol{r}} \times \boldsymbol{m}\right] \\
\boldsymbol{H}^{\mathrm{S}}(\boldsymbol{r})= & \exp \left(\mathrm{i} k_{0} r\right)\left(k_{0}^{2} / 4 \pi r\right) \\
& \cdot\left[Y_{0} \varepsilon_{0}^{-1}(\hat{\boldsymbol{r}} \times \boldsymbol{p})-\hat{\boldsymbol{r}} \times(\hat{\boldsymbol{r}} \times \boldsymbol{m})\right] .
\end{aligned}
$$

Let the static approximations of the scattered fields be expressed as the gradients of potential functions, viz.

$$
\begin{aligned}
E_{0}^{s} & =\lim _{k_{0} \rightarrow 0} E^{s}=-\nabla \Phi_{e}^{s}, \\
\boldsymbol{H}_{0}^{s} & =\lim _{k_{0} \rightarrow 0} \boldsymbol{H}^{s}=-Y_{0} \nabla \Phi_{m}^{s}
\end{aligned}
$$

and in terms of Cartesian coordinates $x_{i}(i=1,2,3)$ let

$\Phi_{e}^{s}=\sum_{i=1}^{3}\left(\hat{\boldsymbol{a}} \cdot \hat{\boldsymbol{x}}_{i}\right) \Phi_{e_{i}}^{s}, \quad \Phi_{m}^{s}=\sum_{i=1}^{3}\left(\hat{\boldsymbol{b}} \cdot \hat{\boldsymbol{x}}_{i}\right) \Phi_{m_{i}}^{s}$.

By expressing the zeroth order (in $k_{0}$ ) fields inside $\mathrm{B}$ in terms of interior electric and magnetic potentials in an analogous manner, the dipole moments can be written as [4]

$\boldsymbol{p}=\varepsilon_{0} \overline{\bar{P}} \cdot \hat{a}, \quad \boldsymbol{m}=-Y_{0} \overline{\bar{M}} \cdot \hat{b}$

where $\overline{\bar{P}}$ and $\overline{\bar{M}}$ are the electric and magnetic polarizability tensors. $\overline{\bar{P}}$ and $\overline{\bar{M}}$ are functions, respectively, of the relative permittivity $\varepsilon_{r}$ and relative permeability $\mu_{r}$ of the dielectric, and of the geometry of the body, but are independent of the direction and polarization of the incident field. For any given incident plane wave, these tensors specify the dipole moments and hence the farzone scattered fields in the Rayleigh region.

The tensor elements $P_{i j}$ and $M_{i j}(i, j=1,2,3)$ are expressible as weighted surface integrals of the electrostatic and magnetostatic potentials, and from the boundary conditions on the potentials at the surface of the body it is found [5] that $\overline{\bar{P}}$ and $\overline{\bar{M}}$ are special cases of a general polarizability tensor $\overline{\bar{X}}(\tau)$ in terms of which

$\overline{\bar{P}}=\overline{\bar{X}}\left(\varepsilon_{r}\right), \quad \overline{\bar{M}}=-\overline{\bar{X}}\left(\mu_{r}\right)$.

It is remarked that for a perfectly conducting body $\varepsilon_{r}=\infty$ and $\mu_{r}=0$, and the $\overline{\bar{P}}$ and $\overline{\bar{M}}$ computed by Kleinman and Senior $[6,7]$ for a number of metallic shapes therefore yield $\overline{\bar{X}}(\tau)$ for these two extreme values of $\tau$. The general polarizability tensor is a function only of the geometry and a material parameter $\tau$ of the body, and for real $\tau$ the tensor is real and symmetric, having at most six independent elements. For an arbitrary homogeneous isotropic body the tensor elements are given by [5]

$X_{i j}=(1-\tau) \int_{\mathrm{B}} \hat{\boldsymbol{n}} \cdot \hat{\boldsymbol{x}}_{i} \Phi_{j} d S$

where $\hat{\boldsymbol{n}}$ is the outward unit vector normal to $\mathrm{B}$, and $\Phi_{j}$ is a total potential exterior to B. (The subscripts $e$ and $m$ 
used to denote the electrostatic and magnetostatic potentials may now be dropped.) The potentials $\Phi_{j}(j$ $=1,2,3$ ) are solutions of the integral equations

$\Phi_{j}(\boldsymbol{r})=\frac{-2}{1+\tau} x_{j}+\frac{1-\tau}{1+\tau} \frac{1}{2 \pi} \int_{\mathbf{B}} \Phi_{j}\left(\boldsymbol{r}^{\prime}\right) \frac{\partial}{\partial n^{\prime}}\left(\frac{1}{R}\right) d S^{\prime}$,

where $\boldsymbol{r}$ is a position vector terminating on $\mathrm{B}, R=\left|\boldsymbol{r}-\boldsymbol{r}^{\prime}\right|$ and the integration is with respect to the primed variables. An alternate representation in terms of the normal derivatives of the potentials can be derived and is

$X_{i j}=\frac{1-\tau}{\tau} \int_{\mathrm{B}} x_{i} \frac{\partial \Phi_{j}}{\partial n} d S$

where

$$
\begin{aligned}
\frac{\partial \Phi_{j}(\boldsymbol{r})}{\partial n}= & \frac{-2 \tau}{1+\tau} \hat{\boldsymbol{n}} \cdot \hat{\boldsymbol{x}}_{j} \\
& +\frac{1-\tau}{1+\tau} \frac{1}{2 \pi} \int_{\mathrm{B}} \frac{\partial \Phi\left(\boldsymbol{r}^{\prime}\right)}{\partial n^{\prime}} \frac{\partial}{\partial n}\left(\frac{1}{R}\right) d S^{\prime} .
\end{aligned}
$$

Although the kernels appearing in the above integral equations are in general weakly singular, it can be shown [8] that the Fredholm alternative remains valid. Examination of the associated homogeneous integral equations obtained by setting the first term on the righthand side equal to zero in (8) and (10) shows that their eigenvalues are all real and greater than or equal to zero in absolute value. Hence (8) and (10) possess unique solutions for all complex $\tau$ and for real $\tau$ greater than zero. It is remarked however that (8) proves more desirable for numerical purposes and the representation in terms of the potentials rather than their normal derivatives is used hereafter. For a body of arbitrary shape the tensor $\overline{\bar{X}}(\tau)$ is then completely specified by the solution of at most the three integral equations (8) with $j=1,2,3$.

\section{Application to a Rectangular Parallelepiped}

A geometry for which the computation of the tensor elements is relatively straightforward is that of a rectangular parallelepiped occupying the region $-a$ $\leqq x_{1} \leqq a,-b \leqq x_{2} \leqq b,-c \leqq x_{3} \leqq c$. The symmetry of the body about the three mutually perpendicular planes $x_{i}$ $=0(i=1,2,3)$ gives rise to corresponding symmetries among the potentials, viz

$$
\begin{aligned}
\Phi_{1}\left(x_{1}, x_{2}, x_{3}\right) & =-\Phi_{1}\left(-x_{1}, x_{2}, x_{3}\right) \\
& =\Phi_{1}\left(x_{1},-x_{2}, x_{3}\right) \\
& =\Phi_{1}\left(x_{1}, x_{2},-x_{3}\right)
\end{aligned}
$$

with analogous relations for $\Phi_{2}$ and $\Phi_{3}$. Making the change of variable $\Psi_{i}=(1-\tau) \Phi_{i}$ the tensor elements are then

$X_{i i}=8 \int_{\mathbf{S}_{i}} \Psi_{i}\left(x_{1}, x_{2}, x_{3}\right) d S_{i}$,

$X_{i j}=0 \quad(i \neq j)$

where the surfaces $S_{i}$ are given by

$\mathrm{S}_{1}: x_{1}=a, \quad 0 \leqq x_{2} \leqq b, \quad 0 \leqq x_{3} \leqq c$

$\mathrm{S}_{2}: 0 \leqq x_{1} \leqq a, \quad x_{2}=b, \quad 0 \leqq x_{3} \leqq c$

$\mathrm{S}_{3}: 0 \leqq x_{1} \leqq a, 0 \leqq x_{2} \leqq b, \quad x_{3}=c$

and the potentials $\Psi_{i}$ are solutions of

$\frac{1+\tau}{1-\tau} \Psi_{i}(r)=-2 x_{i}+\frac{1}{2 \pi} \int_{\mathrm{B}} \Psi_{i}\left(r^{\prime}\right) \frac{\partial}{\partial n^{\prime}}\left(\frac{1}{R}\right) d S^{\prime}$.

The tensor is therefore diagonal with at most three independent elements, and will in general require the solution of the three integral equations (14) with $i=1,2,3$. In the special case of a rectangular parallelepiped of square cross section (for example $a=b$ ) the additional symmetry yields

$\Phi_{1}\left(x_{1}, x_{2}, x_{3}\right)=\Phi_{2}\left(x_{2}, x_{1}, x_{3}\right)$

implying $X_{11}=X_{22}$. Since the tensor now has only two independent elements, it will be necessary to solve only two of the integral equations. Similarly, for a cube $(a=b$ $=c$ ) it is found that $X_{11}=X_{22}=X_{33}$ and the solution of one integral equation is sufficient to completely specify the tensor.

Whereas (14) requires the integration to be carried out over the entire surface of the body, it is desirable for numerical purposes to limit the integration to a smaller portion of the surface. Noting that

$\frac{\partial}{\partial n^{\prime}}\left(\frac{1}{R}\right)=\hat{\boldsymbol{n}}^{\prime} \cdot \frac{\boldsymbol{r}-\boldsymbol{r}^{\prime}}{\left|\boldsymbol{r}-\boldsymbol{r}^{\prime}\right|^{3}}$

and exploring the symmetry relations among the potentials it is found that (14) can be written as

$\frac{1+\tau}{1-\tau} \Psi_{i}(\boldsymbol{r})=-2 x_{i}+\frac{1}{2 \pi} \sum_{j=1}^{3} \int_{\mathrm{S}_{j}} \Psi_{i}\left(\boldsymbol{r}^{\prime}\right) K_{i j}\left(\boldsymbol{r}, \boldsymbol{r}^{\prime}\right) d S_{j}^{\prime}$. 
The kernels $K_{i j}$ in (17) are given by

$$
\begin{aligned}
& K_{i j}\left(x_{1}, x_{2}, x_{3} ; x_{1}^{\prime}, x_{2}^{\prime}, x_{3}^{\prime}\right) \\
& =\sum_{\eta_{1}= \pm 1} \sum_{\eta_{2}= \pm 1} \sum_{\eta_{3}= \pm 1} \eta_{i} \eta_{j}\left(x_{j}+\eta_{j} x_{j}^{\prime}\right) \\
& \cdot\left[\left(x_{1}+\eta_{1} x_{1}^{\prime}\right)^{2}+\left(x_{2}+\eta_{2} x_{2}^{\prime}\right)^{2}+\left(x_{3}+\eta_{3} x_{3}^{\prime}\right)^{2}\right]^{-3 / 2}
\end{aligned}
$$

where $\left(x_{1}, x_{2}, x_{3}\right)$ and $\left(x_{1}^{\prime}, x_{2}^{\prime}, x_{3}^{\prime}\right)$ are the coordinates of the observation and integration points on $\mathrm{B}$, respectively. In particular it is observed that the righthand side of (16) is identically zero whenever $r$ and $\boldsymbol{r}^{\prime}$ lie on the same surface of the body, thus removing the singularity at $r=r^{\prime}$. Hence it is understood that all terms in (18) containing $x_{j}-x_{j}^{\prime}$ are identically zero whenever $x_{j}=x_{j}^{\prime}$, and the solution of (17) by the moment method requires no special treatment of "self cell" contributions to the integrals. Dividing the surfaces of integration into sampling cells and performing a term by term integration over each cell we are faced with integrals of the form

$\int_{\delta_{1}}^{\delta_{2} \varepsilon_{1}} \frac{\Psi_{2}}{\left[(\xi \pm \xi)^{2}+\left(\beta \pm \beta^{\prime}, \gamma^{\prime}\right)(\alpha \pm \xi) d \beta^{\prime} d \gamma^{\prime}\right.}$

where, for example, for a cell on $\mathrm{S}_{1}, \alpha=x_{1}, \beta=x_{2}, \gamma=x_{3}$, $\xi=x_{1}^{\prime}, \beta^{\prime}=x_{2}^{\prime}, \gamma^{\prime}=x_{3}^{\prime}$ and the cell occupies the surface $x_{1}^{\prime}=a, \varepsilon_{1} \leqq x_{2}^{\prime} \leqq \varepsilon_{2}, \delta_{1} \leqq x_{3}^{\prime} \leqq \delta_{2}$. If $\Psi_{i}$ is assumed constant over the surface of the cell, the remaining integration can be performed analytically. Letting

$$
\begin{aligned}
& f\left(\alpha, \beta, \gamma, \xi, \varepsilon_{1}, \varepsilon_{2}, \delta_{1}, \delta_{2}\right) \\
& =\int_{\delta_{1}}^{\delta_{2}} \int_{\varepsilon_{1}}^{\varepsilon_{2}} \frac{(\alpha-\xi) d \beta^{\prime} d \gamma^{\prime}}{\left[(\alpha-\xi)^{2}+\left(\beta-\beta^{\prime}\right)^{2}+\left(\gamma-\gamma^{\prime}\right)^{2}\right]^{3 / 2}} \\
& =\sum_{i, j=1}^{2}(-1)^{i+j} \tan ^{-1}\left\{\frac{\left(\beta-\varepsilon_{i}\right)\left(\gamma-\delta_{j}\right)}{\left[(\alpha-\xi)^{2}+\left(\beta-\varepsilon_{i}\right)^{2}+\left(\gamma-\delta_{j}\right)^{2}\right]^{1 / 2}}\right\}
\end{aligned}
$$

it is found that

$$
\begin{aligned}
& \int_{\delta_{1}}^{\delta_{2}} \int_{\varepsilon_{1}}^{\varepsilon_{2}} K_{11}\left(x_{1}, x_{2}, x_{3} ; x_{1}^{\prime}, x_{2}^{\prime}, x_{3}^{\prime}\right) d S_{1}^{\prime} \\
& =\sum_{\beta= \pm x_{2}} \sum_{\gamma= \pm x_{3}} \sum_{\xi= \pm a} f\left(x_{1}, \beta, \gamma, \xi, \varepsilon_{1}, \varepsilon_{2}, \delta_{1}, \delta_{2}\right)
\end{aligned}
$$

with similar expressions for the other integrals. It is therefore evident that by dividing the surfaces of integration into a total of $n$ sampling cells and letting $q_{m}(m=1,2, \ldots, n)$ represent the coordinates of the center of the $m^{\text {th }}$ cell, (17) can be approximated by a system of nonhomogeneous linear algebraic equations. Expressed in matrix form

$\frac{1+\tau}{1-\tau}\left[\Psi_{i}\left(q_{m}\right)\right]=-2\left[x_{i}\left(q_{m}\right)\right]+(1 / 2 \pi)\left[A_{i}\right]\left[\Psi_{i}\left(q_{m}\right)\right]$,

where $\left[A_{i}\right]$ is an $n \times n$ square matrix whose elements are obtained by integration of the appropriate kernel.

It is also possible to obtain a numerical solution of (17) by assuming the entire integrand to be constant over the surface of a sampling cell. This is the method used by Edwards and van Bladel [9] in their investigation of the perturbation of a uniform electrostatic field by a dielectric cube, and leads to a matrix equation of the same form as (21), but with the elements of the matrix $\left[A_{i}\right]$ obtained by evaluating the kernels using the center coordinates of the observation and integration cells. Computer programs have been written for solving (17) by both techniques, and we have found the program incorporating the analytical integration of the kernels to be far superior from the standpoint of numerical convergence. The program requires fewer sampling cells to achieve the same degree of accuracy, and hence reduces substantially the expense of the computations. In addition, the analytical integration of the kernels simplifies the task of selecting the configuration and distribution of the sampling cells since we are now sampling only the potential $\Psi_{i}$, which is in general a slowly varying function over the surface of the body. Having thus determined the value of $\Psi_{i}$ over each sampling cell, the integration in (12) can be replaced by a finite summation and the tensor elements computed using

$X_{i i}=\sum_{\mathrm{s}_{i}} \Psi_{i}\left(q_{m}\right) \sigma_{m}$,

where $\sigma_{m}$ is the surface area of the $m^{\text {th }}$ cell.

\section{Numerical Results}

The general polarizability tensor $\overline{\bar{X}}(\tau)$ has been computed for rectangular parallelepipeds of square cross section $(a=b)$ having length-to-width ratios $(1 / \mathrm{w})$ from 0.1 to 10 and $\tau$ ranging from zero to infinity. The normalized tensor elements $X_{11}(\tau) / V\left(=X_{22}(\tau) / V\right)$ and $X_{33}(\tau) / V$ are plotted as functions of $\tau$ in Figs. 1 and 2, respectively, with $l / \mathrm{w}$ shown as a parameter. The data were obtained using 75 to 100 sampling cells over the surface of integration in (17) and are accurate to within about one percent, with the maximum error occurring at the extreme values of $\tau$ and $l / w$. Both tensor elements are increasing functions of $\tau$, with $X_{i i}(1)=0$, and for fixed $\tau$ it is seen that as $l /$ w increases, $X_{33}(\tau) / V$ increases 


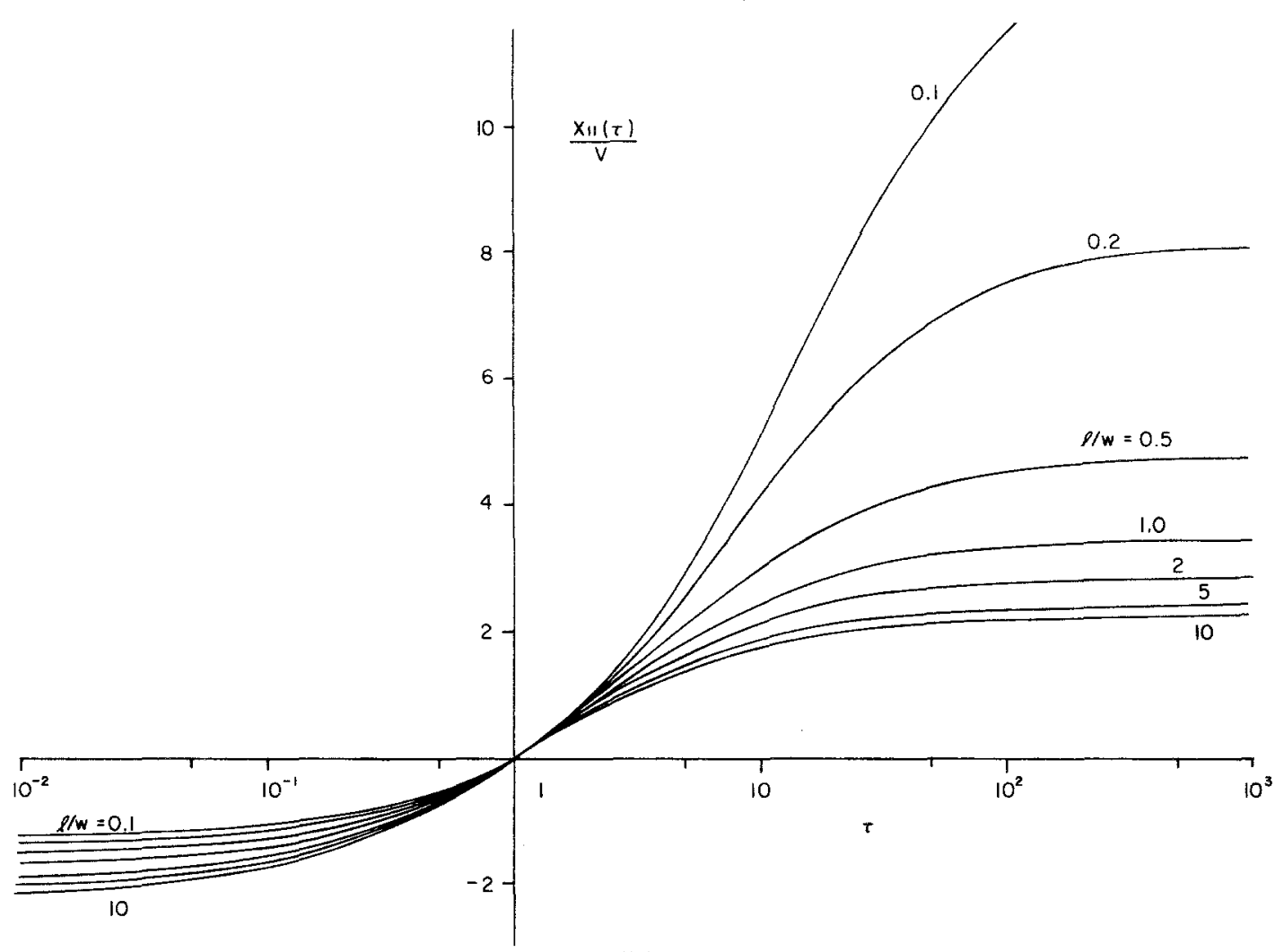

Fig. 1. $X_{11}(\tau) / V$ as a function of $\tau$ for a rectangular parallelepiped of square cross section $(a=b)$

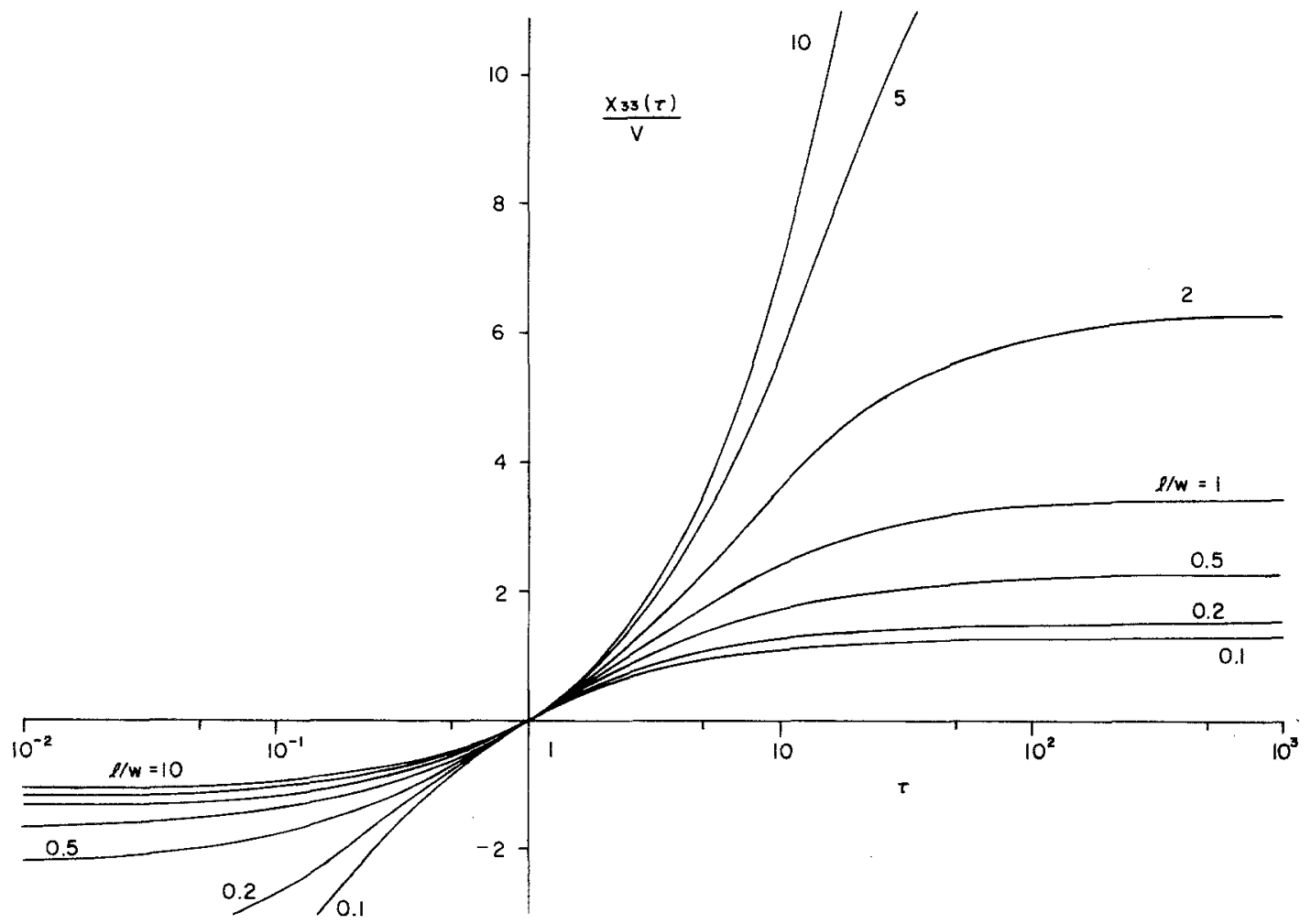

Fig. 2. $X_{33}(\tau) / V$ as a function of $\tau$ for a rectangular parallelepiped of square cross section $(a=b)$ 
Table 1. Tensor elements for a perfectly conducting rectangular parallelepiped

\begin{tabular}{rcccc}
\hline$l / \mathrm{w}$ & $X_{11}(0)$ & $X_{33}(0)$ & $X_{11}(\infty)$ & $X_{33}(\infty)$ \\
\hline 0.1 & -1.19 & -6.44 & 13.3 & 1.26 \\
0.2 & -1.27 & -3.87 & 8.23 & 1.53 \\
0.5 & -1.47 & -2.26 & 4.79 & 2.26 \\
1.0 & -1.65 & -1.65 & 3.54 & 3.54 \\
2.0 & -1.87 & -1.36 & 2.86 & 6.37 \\
5.0 & -2.09 & -1.15 & 2.40 & 17.3 \\
10.0 & -2.18 & -1.08 & 2.26 & 43.6 \\
\hline
\end{tabular}

whereas $X_{11}(\tau) / V$ decreases. We also remark that for a specific body, i.e. fixed $\tau$ and $l / \mathrm{w}$, the ratio $X_{33} / X_{11}$ is important in connection with the polarization characteristics of the scattered fields. For the data presented here, $X_{33} / X_{11}$ varies all the way from 0.1 to 20 , showing the general inadequancy of the spherical model $\left(X_{33} / X_{11}=1\right)$ used in most current theories of scattering by aerosols.
Using (5) and (6), the tensor $\overline{\bar{X}}(\tau)$ evaluated at the extreme values of $\tau=\infty$ and $\tau=0$ yields, respectively, the electric and magnetic dipole moments of a perfectly conducting body. Since these are of particular interest in many applications, the corresponding tensor elements are listed in Table 1. Although there are no parallelepipeds for which exact analytical expressions for the tensor elements are known, there is one test which can be applied to the numerical results. It has been proved by Schiffer [10] that of all perfectly conducting bodies of convex shape, the sphere possesses the minimum average polarizability. Since the elements of the polarizability tensor for a dielectric sphere are

$X_{i i}(\tau)=3 V(\tau-1) /(\tau+2)$

implying $X_{i i}(0)=-3 V / 2$ and $X_{i i}(\infty)=3 V$, this leads immediately to the isoperimetric inequalities

$1 / 3 \sum_{i=1}^{3} X_{i i}(\infty) \geqq 3 V, \quad 1 / 3 \sum_{i=1}^{3} X_{i i}(0) \leqq 3 V / 2$.

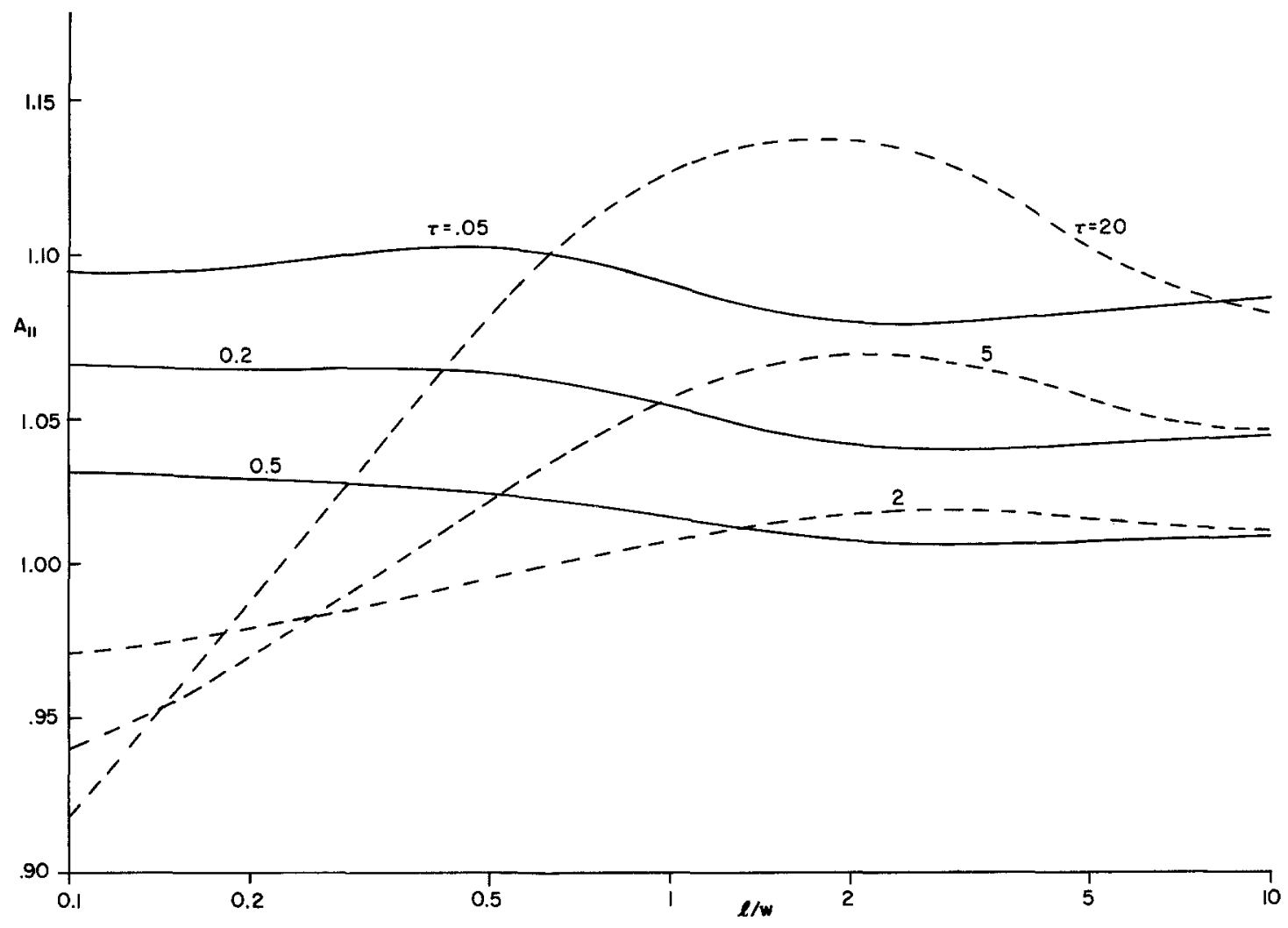

Fig. 3. The tensor element $X_{11}(\tau)$ normalized to that of a spheroid of same volume and material parameter $\tau . A_{11}=X_{11}$ (rectangular parallelepiped) $/ X_{11}$ (spheroid) 
For a rectangular parallelepiped having $X_{11}=X_{22},(24)$ may be written as

$2 X_{11}(\infty) / V+X_{33}(\infty) / V>9$

$2 X_{11}(0) / V+X_{33}(0) / V<-9 / 2$

which are satisfied by the data in Table 1 .

As a part of our general study of low frequency scattering from dielectric particles, the polarizability tensor has also been computed for a number of rotationally symmetric bodies [11]. In particular we have noted that the tensor elements of spheroids and right circular cylinders, as functions of $\tau$ and $l / w$, have substantially the same form as those in Figs. 1 and 2. The tensor elements $X_{11}(\tau) / V$ and $X_{33}(\tau) / V$ computed for rectangular parallelepipeds of square cross section have been normalized to those of a spheroid of equal length-to-width ratio and material parameter $\tau$ and are plotted in Figs. 3 and 4, respectively. The maximum difference is no more than sixteen percent. Similarly, in Figs. 5 and 6 the values of $X_{11} / V$ and $X_{33} / V$ have been normalized to those of the corresponding right circular cylinder, and in this case the maximum difference is no more than ten percent. If we think of Figs. 5 and 6 as representing a comparison between the tensor elements of square and circular cylinders of the same material and having equal length-to-width ratios, then it is apparent (since $X_{i i}(\tau)<0$ for $0 \leqq \tau<1$ ) that

$\frac{X_{11}}{V}\left(\begin{array}{c}\text { square } \\ \text { cylinder }\end{array}\right) \lessgtr \frac{X_{11}}{V}\left(\begin{array}{c}\text { circular } \\ \text { cylinder }\end{array}\right)$

$\frac{X_{33}}{V}\left(\begin{array}{c}\text { square } \\ \text { cylinder }\end{array}\right)<\frac{X_{33}}{V}\left(\begin{array}{c}\text { circular } \\ \text { cylinder }\end{array}\right) \quad(\tau>0)$.

Since a circle can be viewed as the limit of an $n$-sided regular polygon as $n \rightarrow \infty$, it seems reasonable to conjecture that the tensor elements of a cylinder whose cross section is an $n$-sided regular polygon with $n>4$ lie between those of the corresponding square and circular cylinders. The data computed for the square and circular cylinders would then be sufficient to specify the tensor elements of, for example, a hexagonal crystal to within five percent or less.

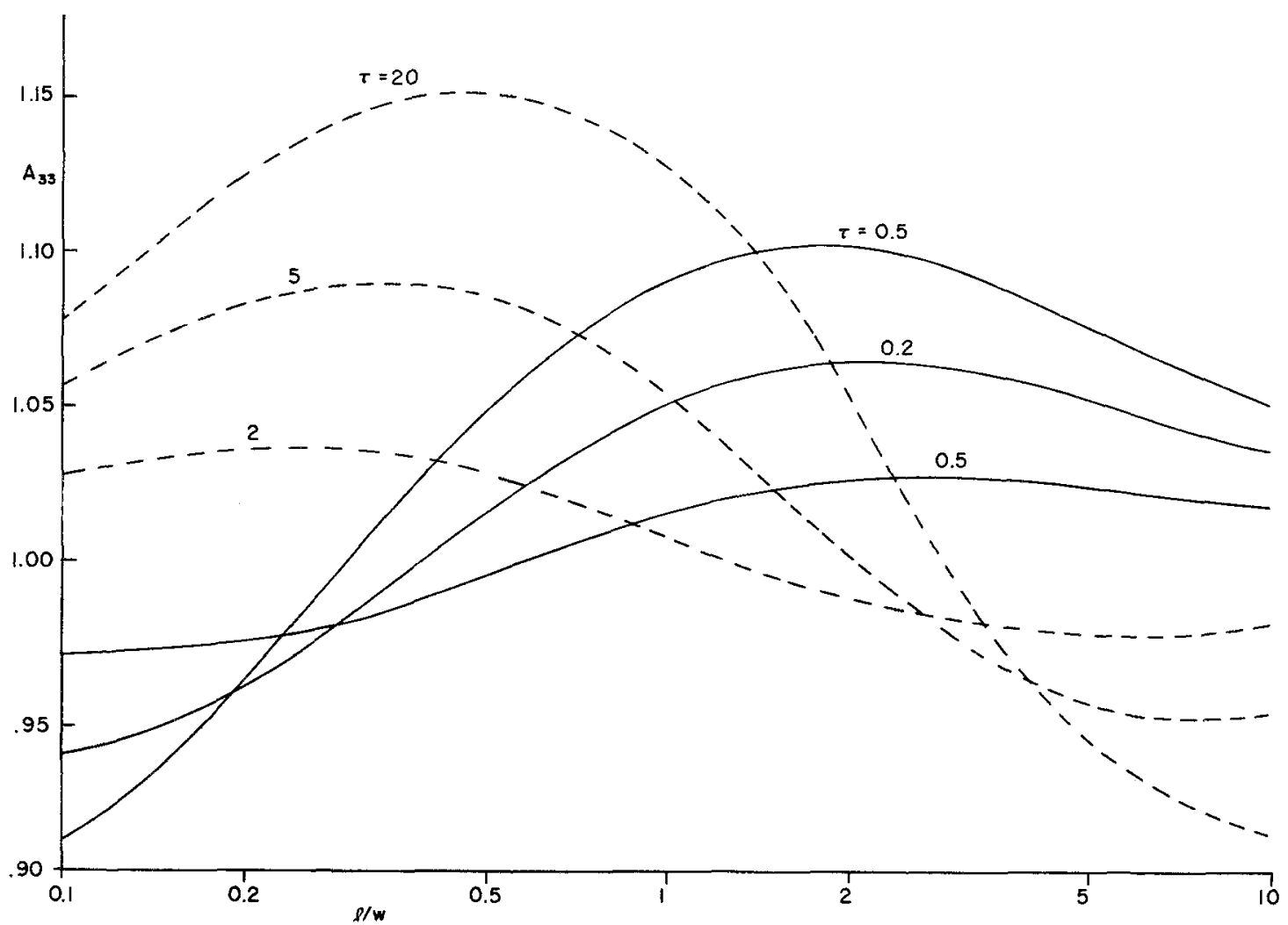

Fig. 4. The tensor element $X_{33}(\tau)$ normalized to that of a spheroid of same volume and material parameter $\tau$. $A_{33}=X_{33}$ (rectangular parallelepiped) $/ X_{33}$ (spheroid) 


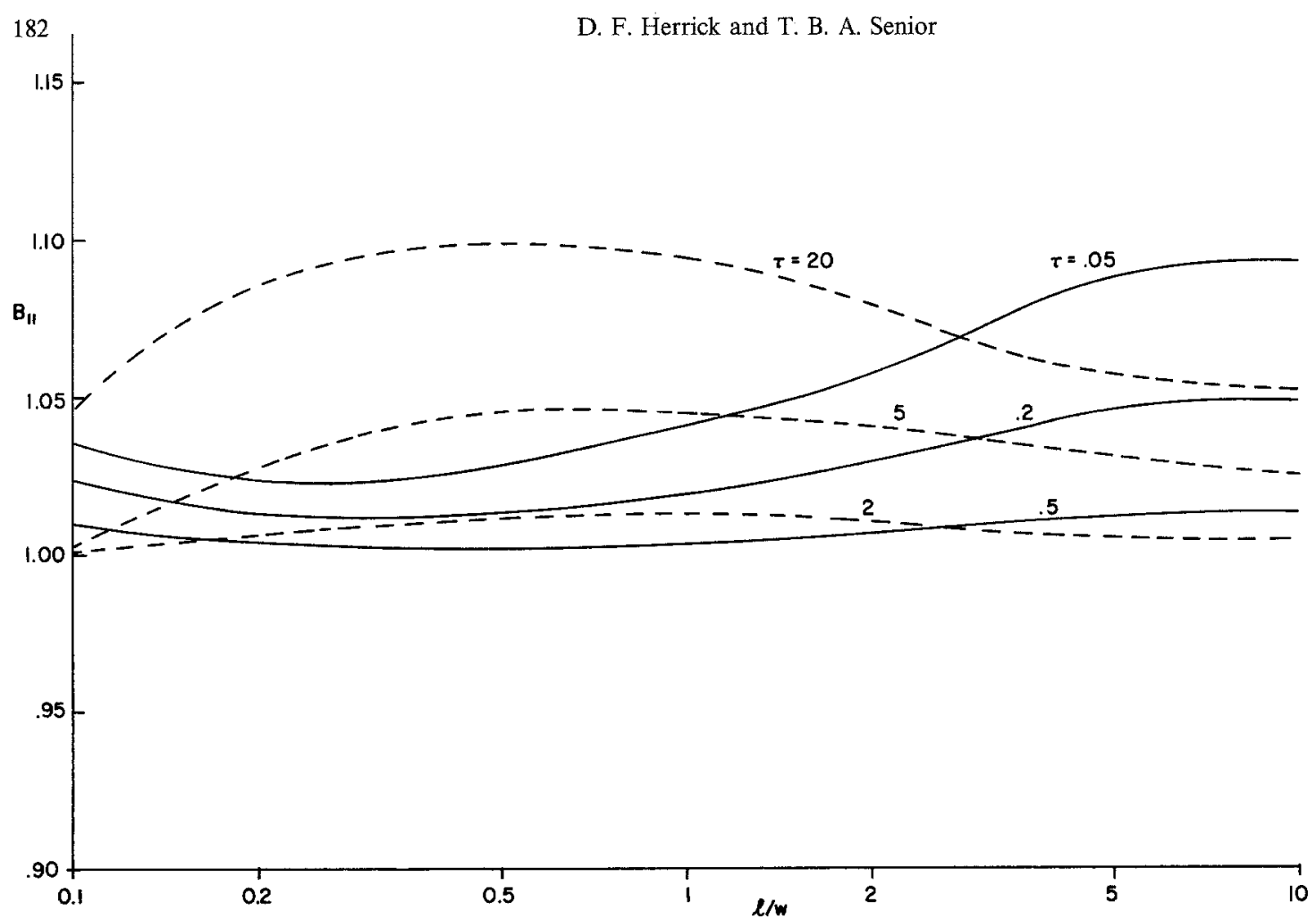

Fig. 5. The tensor element $X_{11}(\tau)$ normalized to that of a right circular cylinder of same volume and material parameter $\tau$. $B_{11}=X_{11}$ (rectangular parallelepiped) $/ X_{11}$ (circular cylinder)

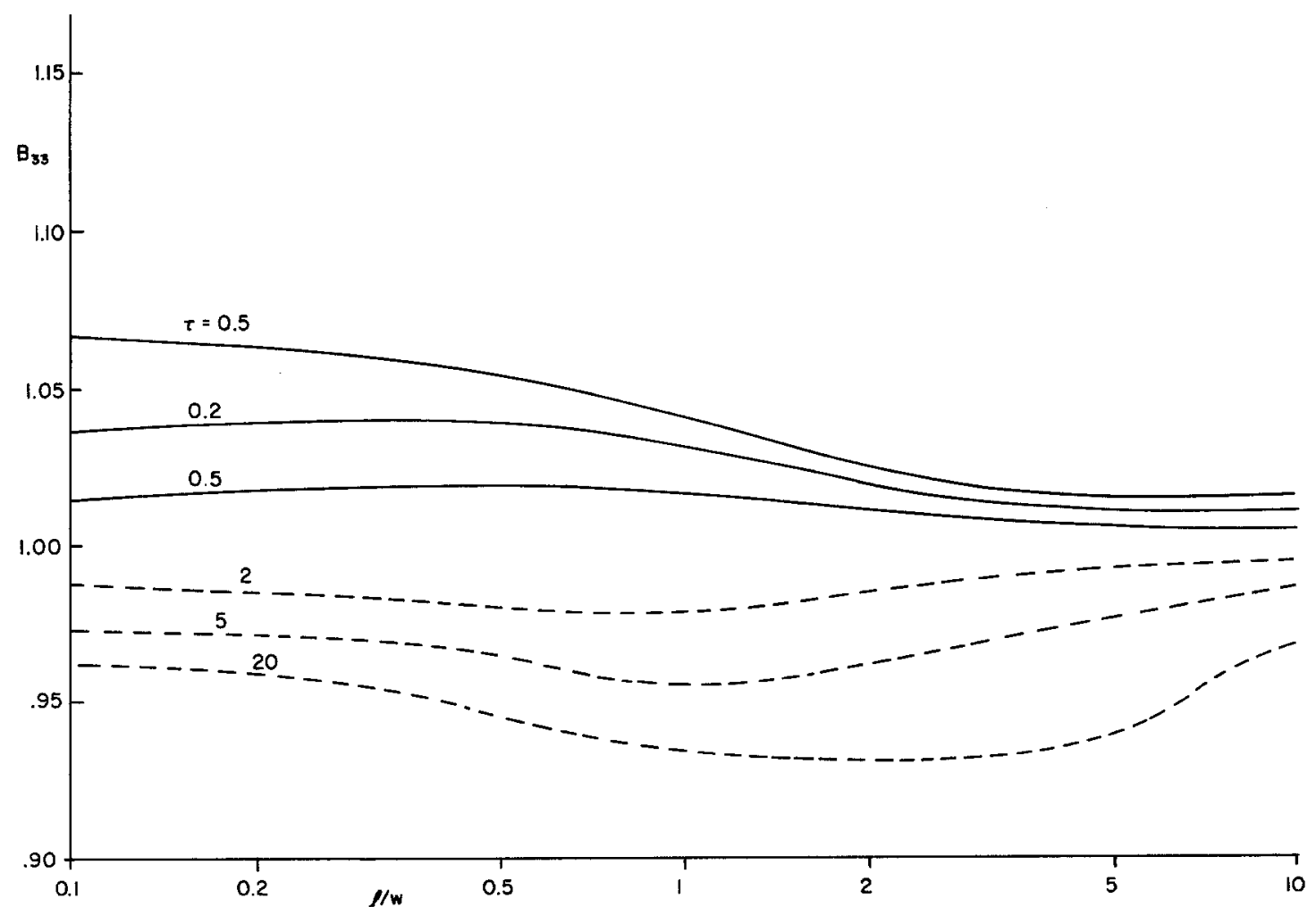

Fig. 6. The tensor element $X_{33}(\tau)$ normalized to that of a right circular cylinder of same volume and material parameter $\tau$. $B_{33}=X_{33}$ (rectangular parallelepiped)/ $X_{33}$ (circular cylinder) 
Acknowledgements. This work was supported by the National Science Foundation Grant ENG 75-03880 and the U.S. Air Force Office of Scientific Research Grant 72-2262. The authors are indebted to Messrs. K. Graves and G. Hopp for their assistance with the computer programming.

\section{References}

1. G.N.Plass, G.W.Kattewar, F.E.Catchings: Appl. Opt. 12, 314 (1973)

2. G.W.Kattewar, G.N.Plass: Appl. Opt. 11, 2851 (1972)

3. A.F.Stevenson: J. Appl. Phys. 24, 1143 (1953)
4. J.B.Keller, R.E.Kleinman, T.B.A.Senior: J. Inst. Maths. Applics. 9, 14 (1972)

5. T.B. A.Senior: Radio Sci. 11, 477 (1976)

6. R.E.Kleinman, T.B.A.Senior: Radio Sci. 7, 937 (1972)

7. R.E.Kleinman, T.B.A.Senior: IEEE Trans. Aerospace and Elect. Syst. 11, 672 (1975)

8. P.R.Garabedian: Partial Differential Equations (John Wiley and Sons, New York 1964) pp 353-361

9. T.W.Edwards, J.Van Bladel: Appl. Sci. Res. 9(B), 151 (1961)

10. M.Schiffer: C.R. Acad. Sci. (Paris) 244, 3118 (1957)

11. D.F.Herrick, T.B.A.Senior: "Low frequency scattering by rotationally symmetric dielectric particles" (to be published) 